\title{
LETTER
}

\section{Groups' Use of Patient Experience Data}

\author{
Beth A. Lown, MD and Julie Rosen, MMHS \\ The Schwartz Center for Compassionate Healthcare, Mount Auburn Hospital, Harvard Medical School, Boston, MA, USA.
}

J Gen Intern Med 26(5):466

DOI: $10.1007 / \mathrm{s} 11606-011-1652-6$

(c) Society of General Internal Medicine 2011

\section{To the Editor:}

We read with interest the findings of Friedberg et al. that although a majority of physician groups reported using patient experience reports to improve group-wide performance, few focused on improving physician-patient communication. ${ }^{1}$ In a recent national poll of 500 physicians and 800 recently hospitalized patients conducted by the Schwartz Center for Compassionate Healthcare (Boston, MA), the majority of patients and physicians agreed that effective communication and emotional support can make a difference in whether a patient lives or dies. ${ }^{1}$ Licensing and accrediting bodies require demonstration of competency in interpersonal and communication skills, and the Patient Protection and Affordable Care Act of 2010 proposes including survey results of patients' experiences of care (including provider communication) among the measures to calculate value-based incentive payments for hospital discharges. ${ }^{2}$

It's unclear why so few quality improvement efforts have included education to improve clinician's patient-centered communication given the increased recognition of its importance. Participants in the study by Friedberg et al. reported sensitivity to low morale among primary care physicians and reluctance to engage physicians' skepticism about their patient experience reports. Changing physicians' communication behaviors is not an easy task. Strong evidence suggests that intensive training interventions, especially with longitudinal reinforcement sessions, can improve clinicians' communication with patients, enable patients to express their concerns and improve patients' satisfaction with care. ${ }^{3,4}$ Such training requires taking time away from practice and implementing systems to support interpersonal communication.

Schwartz Center Rounds, which are offered in more than 200 hospitals, outpatient centers and nursing homes, provide a monthly or bi-monthly forum for clinicians and other team members to discuss the psychosocial and emotional aspects of care and their impact on patients, families and themselves. Attendees report enhanced attention to these issues; better communication with patients, family members, and colleagues; and decreased stress and isolation. ${ }^{5}$ Further research is warranted on interventions designed to improve the capacity of physicians and physician groups to communicate effectively and to provide the emotional support that both patients and physicians believe can make life or death differences.

Compassionate care-defined as patient-centered communication and emotional support of patients-must be measured and valued on par with other clinical process and outcomes measures. Changes in provider reimbursement based in part on patients' experiences of care may provide the impetus for this culture change.

Corresponding Author: Beth A. Lown, MD; The Schwartz Center for Compassionate Healthcare, Mount Auburn Hospital, Harvard Medical School, 205 Portland Street, Boston, MA 02114, USA (e-mail: balown@partners.org).

\section{REFERENCES}

1. Friedberg MW, SteelFisher GK, Karp M, Schneider EC. Physician Groups' Use of Data from Patient Experience Surveys. J Gen Intern Med. Published on-line: 15 December 2010. doi:10.1007/s11606-010-1597-1.

2. Department of Health and Human Services. Centers for Medicare \& Medicaid Services. 42 CFR Parts 422 and 480. Medicare Program; Hospital Inpatient Value-Based Purchasing Program. Federal Register. Vol. 76, No.9. Thursday, January 13, 2011. Proposed Rules. http://www. gpo.gov/fdsys/pkg/FR-2011-01-13/pdf/2011-454.pdf. Accessed February 6, 2011.

3. Rao JK, Anderson LA, Inui TS, Frankel RM. Communication interventions make a difference in conversations between physicians and patients: a systematic review of the evidence. Med Care. 2007;45:340-9.

4. Lewin S, Skea Z, Entwistle VA, Zwarenstein M, Dick J. Interventions for providers to promote a patient-centred approach in clinical consultations. Cochrane Database of Systematic Reviews 2001, Issue 4. Art. No.: CD003267. doi:10.1002/14651858.CD003267.

5. Lown BA, Manning CF. The Schwartz Center Rounds: Evaluation of an Interdisciplinary Approach to Enhancing Patient-Centered Communication, Teamwork, and Provider Support. Acad Med. 2010;85:1073-81. $\overline{1 \text { The Schwartz Center For Compassionate Healthcare. The State of }}$ Compassionate Healthcare in the US. Results of a National Survey of Patients and Doctors. Unpublished data. November, 2010.

Published online February 15, 2011 